\title{
Dose-Dependent Relationship of Physical and Depressive Symptoms with Health-Related Quality of Life in Patients with Heart Failure
}

\author{
Seongkum Heo, PhD, RN [Assistant Professor], \\ University of Arkansas for Medical Sciences, College of Nursing \\ Debra K. Moser, DNSc, RN, FAAN [Professor and Gill Chair of Nursing], \\ University of Kentucky, College of Nursing \\ Susan J. Pressler, PhD, RN [Professor], \\ University of Michigan, School of Nursing \\ Sandra B. Dunbar, DSN, RN [Professor], \\ Emory University, School of Nursing \\ JinShil Kim, PhD, RN [Assistant Professor], \\ Sunchon National University Dept. of Nursing \\ Songthip Ounpraseuth, PhD [Associate Professor], and \\ University of Arkansas for Medical Sciences, Department of Biostatistics \\ Terry A. Lennie, PhD, RN [Professor] \\ University of Kentucky, College of Nursing
}

\section{Abstract}

Background-Patients with heart failure (HF) have poor health-related quality of life (HRQOL). The vast majority of patients have physical symptoms, and about 30 to $40 \%$ have depressive symptoms. The combined effects of physical and depressive symptoms on HRQOL have not been examined fully in HF.

Purposes-To examine the combined effects of physical and depressive symptoms on HRQOL using repeated measures, controlling for covariates (i.e., age, education level, New York Heart Association [NYHA] functional class, financial status, and health perception).

Methods-Patients ( $\mathrm{N}=224,62 \pm 12$ years old, $67 \%$ male, 38\% NYHA functional class III/IV) provided data on physical (Symptom Status Questionnaire) and depressive symptoms (Beck Depression Inventory II) at baseline and HRQOL (Minnesota Living with Heart Failure Questionnaire) at baseline and 12 months. Patients were divided into three groups based on presence of physical and depressive symptoms: a) no symptom group, b) one symptom group

\footnotetext{
Address for correspondence: Seongkum Heo, PhD, RN, Assistant Professor, University of Arkansas for Medical Sciences, College of Nursing, 4301 W. Markham Street \#529, Little Rock, AR 72205, Phone 501-296-1984, sheo@ uams.edu.

Declaration of Conflicting Interests

'The Author(s) declare(s) that there is no conflict of interest'.
} 
(dyspnea or fatigue), and c) two symptoms group (physical and depressive symptoms). Repeated measures ANOVA was used to analyze the data.

Results-The least squares mean scores of baseline and 12-month HRQOL differed significantly in the three groups after controlling for the covariates (26.4 vs. 36.6 vs. 53.1, respectively, all pairwise $\mathrm{p}$ values <.001). There was no time-by-group interaction or time main effect.

Conclusion-Physical and depressive symptoms have a dose-response relationship with HRQOL. Further research is needed to develop and deliver effective interventions to improve physical and depressive symptoms and to determine if improvement in physical and depressive symptoms results in improvement in HRQOL.

\section{Keywords}

Heart failure; quality of life; symptoms; depressive symptoms

\section{Introduction}

Health-related quality of life (HRQOL) is considerably poorer in patients with heart failure (HF) than in patients with other chronic conditions and healthy people of similar ages ${ }^{1,2}$. Poor HRQOL is related to both high hospitalization and high mortality rates in this population ${ }^{3}$. For example, a 10-point decrement in HRQOL score has been found related to a $23 \%$ to $36 \%$ increase in the risk of rehospitalization or mortality ${ }^{3}$. Therefore, improvement in HRQOL is important for patients with $\mathrm{HF}^{4}$.

In patients with HF, there is a high prevalence of physical symptoms, including dyspnea and fatigue ${ }^{5,6}$. Physical symptoms are associated with both hospitalizations and HRQOL in patients with HF. Approximately $92 \%$ to $100 \%$ of ambulatory and hospitalized patients with HF experienced dyspnea ${ }^{7}$. In addition, physical symptoms explained considerable amount of the variance (32\%) in HRQOL in a cross sectional study ${ }^{5}$. In one study ${ }^{8}$, dyspnea and fatigue and their effects on functional status at baseline predicted HRQOL at 3 months. However, the unique long term effects of physical symptoms on HRQOL have not been fully examined in patients with HF. More than $30 \%$ of patients with HF also have depressive symptoms ${ }^{9}$. Depressive symptoms may affect HRQOL through the effects on functional status and physical symptoms. For example, depressed patients with HF compared with nondepressed patients with HF have more limited daily activities and reduced distance in 6minute walk test ${ }^{10}$. Depressive symptoms are also associated with severe physical symptom status ${ }^{8}$. Both functional status and physical symptoms are important factors associating with HRQOL in patients with $\mathrm{HF}^{6,8}$. Thus, physical and depressive symptoms may have a synergic effect on HRQOL in this population. Yet, the associational and long-term effects of these symptoms on HRQOL have not been fully examined. Therefore, the purpose of this study was to examine the combined associational and long-term effects of physical and depressive symptoms on HRQOL. 


\section{Methods}

\section{Design, setting, and sample}

In this longitudinal study, patients with HF were recruited from the outpatient clinics at university-affiliated hospitals and community hospitals in three cities in the United States. In this study, the original purpose was to examine the relationships among body mass index, nutrition, inflammation, symptoms, and HF outcomes, including HRQOL, hospitalizations, and mortality. Inclusion criteria were the following: 1) diagnosis of HF, 2) ability to read and speak English, and 3) optimization of medical therapy (stable on current medication for two clinic visits). The diagnosis of HF was confirmed by clinical signs and symptoms and radiographic evidence through medical record review ${ }^{11}$. Exclusion criteria were the following: 1) myocardial infarction within the previous 3 months, 2) severe cognitive or psychiatric problems that precluded the patient providing informed consent or answering questionnaires, and 3) valvular or postpartum HF etiology. Among 275 patients who were enrolled between December, 2004 and March, 2009, data from 51 patients were excluded: 10 patients withdrew from the study, and 41 patients did not complete the follow-up because the study was closed or had missing data. Thus, data from 224 patients were analyzed. In independent t-tests and Chi-square tests, there were no differences in age, gender, marital status, ethnicity, education level, comorbidities, left ventricular ejection fraction, etiology, and NYHA functional class between the patients who were included and excluded in this analysis.

\section{Measures}

Health-Related Quality of Life-Health-related quality of life was defined as a patient's subjective perception of the physical, psychological, and social effects of HF on daily life ${ }^{12}$. The Minnesota Living with Heart Failure questionnaire was used to assess HRQOL at baseline and 12 months after enrollment. This is the most commonly used instrument to assess HRQOL in this population and has been shown acceptable reliability and validity ${ }^{13-15}$. The instrument includes 21 items with 6 response options (0: no impact of HF on daily life to 5: most negative impact of HF on daily life). The total score is calculated by summing all the ratings, and the possible scores range from 0 to 105, indicating lower scores mean better HRQOL. This instrument has two dimensions: physical and emotional. The physical and emotional dimensions consist of 8 and 5 items, respectively. The reliability of the instrument has been supported in several studies, with Cronbach's alphas greater than . $70{ }^{14,15}$. Construct validity has been supported by factor analyses and the demonstration of hypothesized relationships (e.g., between HRQOL and severity of HF) in several studies ${ }^{14,15}$. In the current study, Cronbach's alphas at baseline and 12-months were .93 and .95 , respectively.

Physical symptoms-Physical symptoms were defined as common physical symptoms related to HF. Physical symptoms were assessed using the Symptom Status questionnaire at baseline. This instrument, a modified version of the Memorial Symptom Assessment ScaleHF assesses the frequency, severity, and distress of seven common HF symptoms: dyspnea during day time and when lying down on the bed, fatigue, chest pain, edema, sleeping difficulty, and dizziness ${ }^{16}$. Patients are asked to indicate whether they have each symptom, 
and if they have a symptom, they are asked to indicate the frequency, severity, and distress of the symptom. Frequency is ranked using 4 response options ranging from 1 (less than once per week) to 4 (nearly daily). Severity is ranked using 4 response options ranging from 1 (slight) to 4 (very much). Distress is ranked using 5 response options ranging from 0 (not at all) to 4 (very much). The total score is calculated by adding all of the ratings. The possible range is 0 to 84 , and lower scores mean better physical symptom status. Reliability of the Memorial Symptom Assessment Scale-HF has been supported by Cronbach's alphas greater than $.70^{16}$. Validity was supported by demonstration of the hypothesized relationship between physical symptoms and HRQOL. In the current study, Cronbach's alpha for the instrument was .93. In the current study, data on the presence of the most common HF symptoms (dyspnea during day time and when lying down on the bed and fatigue) were used to divide those who had physical symptoms and those who did not ${ }^{7}$.

Depressive symptoms-Depressive symptoms were assessed at baseline by the Beck Depression Inventory II, which assesses the severity of cognitive-affective and somatic depressive symptoms ${ }^{17}, 18$. The instrument includes 21 items with four response options. Total scores can range from 0 to 63: scores from 14 to 19 indicate mild depression; scores from 20 to 28 , moderate depression; and scores from 29 to 63 , severe depression ${ }^{18}$. In the current study, patients were divided into two groups: a depressive group (the score 214 ) vs. a non-depressive group (the score $<14$ ) because the cut point indicates at least mild depressive symptoms and has been used as a cut point for depressive symptoms ${ }^{18,19}$. The reliability and validity of this instrument have been supported with several populations ${ }^{17}, 18$. Internal consistency coefficient alphas were greater than $.70^{18}$. Validity was supported by demonstration of hypothesized relationship of this instrument and an anxiety measure ${ }^{17}$, and factor analysis in college students ${ }^{17}$. Cronbach's a in the current study was .91 .

Other variables-Data on socio-demographic characteristics (age, gender, marital status, ethnicity, education level, and financial status) and clinical characteristics (comorbidities, left ventricular ejection fraction, etiology, NYHA functional class, and medication) were collected using standard sociodemographic and clinical questionnaires. Ethnicity was divided into two groups: Caucasian vs. other. Financial status was defined as individuals' perceptions of their income as compared with the demand and assessed using an item on the sociodemographic questionnaire. Response options were: 1) are comfortable; have more than enough to make ends meet; 2) have enough to make ends meet; 3) do not have enough to make ends meet." Validity was supported in our previous study by the significant relationship between financial status and event-free survival and HRQOL ${ }^{20}$. The Charlson Comorbidity Index was used to assess comorbidities ${ }^{21}$. Data on left ventricular ejection fraction, etiology, and medications were examined using the clinical questionnaire through medical record review. New York Heart Association functional class was determined by trained research associates or one of the authors through in-depth patient interviews at baseline. The validity of these instruments has been supported by testing the hypothesized relationships to HRQOL in patients with $\mathrm{HF}^{22}$. Health perception was defined as each individual's overall perception of his or her health status ${ }^{23}$, and assessed using an item from the Medical Outcomes Study SF-36 ${ }^{24}$. Lower scores indicate better health perception. 
Validity of health perception was supported in our study by demonstrating the significant relationship with HRQOL ${ }^{23}$.

Procedure-Approval from appropriate Institutional Review Boards was obtained before data collection. The trained research associates reviewed medical records to identify eligible patients based on the inclusion and exclusion criteria. Written informed consent was obtained from all participants before data collection by the trained research associates or one of the authors. After the consent process, one of the research team members collected baseline data on physical and depressive symptoms, the covariates, baseline HRQOL, and other demographic and clinical characteristics using questionnaires, patient interview, and medical record review. Data on 12-month HRQOL were collected by phone interview with patients.

\section{Data analysis}

Patients were divided into three groups based on the presence or absence of physical and depressive symptoms. The three groups were a no symptom group (no physical symptoms and depressive symptoms), an one symptom group (presence of any type of dyspnea or fatigue) and a two symptom group. There was no fourth group of depressive symptoms only because those who had depressive symptoms also had physical symptoms.

Quantitative data analyses were performed on bivariate and multivariate levels. Initially, differences among study participants' key demographic characteristics and baseline clinical characteristics between the three groups were assessed using appropriate bivariate analyses such as chi-square, analysis of variance (ANOVA), or Kruskal-Wallis.

To test the overall difference between groups, we compared changes in HRQOL among the three groups from baseline to 12-months follow-up. To account for the repeated measurements from each study participant, repeated measures ANOVA was used. The time and group-by-time interactions were included as fixed effects in the model to evaluate how the group means changed from baseline to 12-months follow-up, and how differences between group means changed over time. Various covariance structures were compared among the different repeated ANOVA models based on graphical tools and information criteria such as the restricted maximum likelihood ratio test, the Akaike information criterion, and the Schwarz's Bayesian information criterion. To obtain the most parsimonious model, key sociodemographic and/or clinical variables were included as fixed effects if they were statistically significant at the bivariate baseline analysis level across the three group conditions. All statistical tests were two-tailed with $\mathrm{p}<0.05$ considered statistically significant.

Covariates that are related to HRQOL in the current study were selected based on a tested model and previous literature. In the tested model ${ }^{23}$, age, health perception, and symptoms were related to HRQOL. Age and health perception were used as covariates in the current study. In addition, other variables, including education level, functional status, and financial status were selected as covariates based on prior research $16,25,26$. 


\section{Results}

Twenty seven percent of patients reported at least mild depressive symptoms (Beck Depression Inventory score 214 ). The majority of the patients were categorized in the one symptom group $(n=138,62 \%)$ followed by the two symptom group $(n=61,27 \%)$ and the no symptom group $(n=25,11 \%)$. Both sample demographic and clinical characteristics are presented in Table 1 . The average age of the patients was 62 years old with approximately $67 \%$ were males. Additionally, the majority of them were Caucasian. Among the patient characteristics at baseline, age, education level, financial status, NYHA functional class, and health perception significantly differ across the three symptom groups. The two symptom group compared with the other groups was younger and less educated, had more financial difficulties, experience more functional impairment, and had poor health perception.

The repeated measures ANOVA was used to address basically three questions: how group means differ (i.e., group main effect), how group means change with time (i.e., time main effect), and how differences between group means change over time (i.e., time-by-group interaction) ${ }^{27}$. To control for baseline differences in age, education level, financial status, NYHA functional class, and health perception, these characteristics were included in the longitudinal mixed model as fixed effects. As shown in Figure 1, the analysis resulted in no time-by-group interaction or time main effect. However, group means differ even after adjusting for the other covariates in the model $(\mathrm{p}<0.001)$. More specifically, the least squares mean for the no symptom group was statistically lowest among the three groups (26.4), indicating the best HRQOL among the three groups. The least squares mean difference between the no symptoms and one symptom groups was 10.2. Moreover, there was a statistical difference among the one symptom and two symptom groups with a least squares mean difference of 16.5 (36.6 vs. 53.1) ( $\mathrm{p}<0.001)$. We note that in the repeated measures model, the only covariate which remained statistically significant was health perception $(\mathrm{p}<0.001)$.

\section{Discussion}

The findings of the current study demonstrated the dose-dependent relationships of physical and depressive symptoms on HRQOL in patients with HF. The HRQOL scores in the three groups significantly differed, even after control covariates. The least squares mean scores indicate that more symptoms were connected to more reduction of HRQOL status. This dose-dependent relationship of physical and depressive symptoms on HRQOL clearly shows the significant effects of depressive symptoms as well as physical symptoms on HRQOL in patients with HF. However, depressed patients with HF are not fully recognized and treated ${ }^{28,29}$. Furthermore, not many HF interventions have been focused on managing both physical and depressive symptoms ${ }^{30,31}$. Thus, clinicians who work with HF patients need to assess and manage not only physical symptoms but also depressive symptoms to improve HRQOL. In addition, researchers and clinicians need to develop and deliver comprehensive interventions targeting both physical and depressive symptoms to patients with HF.

The current study demonstrated the long-term consistent relationships of physical and depressive symptoms to HRQOL in patients with HF. Prior studies examined either of the 
relationships between physical symptoms and HRQOL or the relationships between depressive symptoms and HRQOL in patients with HF, but not the combined long-term effects on HRQOL. In several cross-sectional studies $5,6,16$, physical symptoms regardless of the measures used (frequency and severity, number of symptoms, or symptom burden) were associated with HRQOL. One of our previous studies found a short-term (3 month) predictive effect of physical symptoms on HRQOL ${ }^{8}$. Symptom status in the study was measured by Dyspnea-Fatigue Index that reflects not only the symptom status but also the effects on functional status. The significance of depressive symptoms on HRQOL in the current study was consistent with the findings in previous studies 9,32 . The current study showed the unique long-term effects of physical symptoms on HRQOL as well as the combined effects with depressive symptoms on HRQOL.

Physical symptoms may affect HRQOL directly and also through the effects on functional status that is one important factor associating with HRQOL in patients with $\mathrm{HF}^{6}, 16$. Depressive symptoms may affect HRQOL directly and through the effects on functional status and also symptoms. For instance, daily activities and walking distance were more reduced in depressed patients with HF compared with non-depressed patients ${ }^{10}$. In addition, the symptom status was more severe in more depressed patients compared with less depressed patients 5,8 . This may explain why the least squares mean difference between the one and two symptom groups was bigger than that between the no and one symptom groups (16.5 vs. 10.2). This also may explain why the two symptom group experienced the worst HRQOL among the three groups.

In the current study, HRQOL score in each group did not change over time. In the one symptom and two symptom groups, baseline HRQOL was poor, especially in the two symptom group, and the poor HRQOL status remained over time. In the current study, those patients who did not have optimized medications were excluded. As a result, patients who had relatively stable condition were included. The findings of the current study are consistent with the finding of a prior study of patients admitted for HF, in which HRQOL was improved in the first month after discharge from hospital, but was unchanged after that ${ }^{33}$. This fact is important in this population because improvement in HRQOL is as important as longer survival in patients with $\mathrm{HF}^{4}$. No changes in HRQOL despite treatment may imply that the treatment of physical and depressive symptoms was not effective enough to improve HRQOL over time in this sample. We did not measure physical and depressive symptoms at 12-months, so changes in symptoms in this sample between baseline and 12 months cannot be known. However, no improvement in HRQOL scores between baseline and 12-months in the one and two symptom groups suggests that more effective interventions need to be provided to improve HRQOL in patients with HF.

Some intervention studies have targeted improvement of both physical and depressive symptoms and have demonstrated positive effects. For example, a mindfulness-based psycho-educational support group intervention ${ }^{30}$, and a home-based comprehensive management program decreased depressive symptoms and improved physical symptoms ${ }^{31}$. In the mindfulness-based psycho-educational support group intervention ${ }^{30}$, mindfulness based stress reduction, coping skills training, and expressive support group discussion were provided. Clearly, to improve physical and depressive symptom status, and, in turn, 
HRQOL, we need to focus on comprehensive self-care, including psychosocial support. In addition, researchers need to determine whether improvement in physical and depressive symptoms results in improvement in HRQOL.

Comprehensive interventions have to be provided to especially HF patients who have both physical and depressive symptoms. In the current study, the two symptom group compared with the other two groups was younger, received less education, and had more financial difficulties and poorer health perception. The reason for younger age in the two symptom group compared with the other two groups is depressive symptoms. If age has been associated with depressive symptoms, younger age was associated with more severe depressive symptoms in patients with cardiac diseases, including $\mathrm{HF}^{34,35}$. Younger age was also associated with poor HRQOL ${ }^{36}$. Younger people may have more psychological distress due to HF because it is difficult for them to fulfill their various responsibilities for their family and to participate in social activities that their peers usually can do, and that they were used to do. This psychological distress in younger patients may be related to depressive symptoms and the worst HRQOL in the two symptom group. Thus, clinicians need to assess and manage depressive symptoms in relatively young HF patients. Poor social status was connected to depressive symptoms in the current study, and it is also associated with poor event-free survival ${ }^{20}$. Thus, clinicians need to work with these patients more closely to reduce medical cost and provide information about available social support. For example, physicians or nurses can prescribe medication that is cheaper than other medications, but has similar effects. The two symptom group had poor health perception that was the only covariate that predicted HRQOL in the current study. Health perception was also associated with HRQOL in a prior HF study ${ }^{23}$. Thus, more studies are needed to assess factors affecting health perception and to develop interventions targeting improvement in health perception.

There are some limitations in this study. Physical and depressive symptoms were assessed based on self-report. Thus, the actual physical and depressive symptom status may be different from the status reported in the current study. The mean age of the sample in the current study was relatively young. In older patients with HF, symptom status and the effects on HRQOL may differ from those reported in the current study. Nonetheless, the findings of this study provide important information about the combined long-term effects of physical and depressive symptoms on HRQOL in patients with HF.

\section{Conclusion}

Physical and depressive symptoms have a dose-response relationship with HRQOL. Thus, clinicians need to assess and treat depressive symptoms as well as physical symptoms to improve HRQOL effectively. Clinicians and researchers need to develop and deliver interventions focused on improvement in both physical and depressive symptoms to improve HRQOL. Researchers need to examine whether improvement in physical and depressive symptoms results in improvement in HRQOL in patients with HF. 


\section{Acknowledgments}

Funding for this study came from an American Heart Association Postdoctoral Fellowship to Seongkum Heo, National Institutes of Health (NIH), National Institute of Nursing Research (NINR) R01 NR009280 to Terry Lennie, and Center grant, NIH, NINR, 1P20NR010679 to Debra Moser.

\section{Abbreviations}

$\begin{array}{ll}\text { ANOVA } & \text { Analysis of variance } \\ \text { HF } & \text { Heart failure } \\ \text { HRQOL } & \text { health-related quality of life } \\ \text { NIH } & \text { National Institutes of Health } \\ \text { NINR } & \text { National Institute of Nursing Research } \\ \text { NYHA } & \text { New York Heart Association }\end{array}$

\section{References}

1. Dixon T, Lim LL, Oldridge NB. The MacNew heart disease health-related quality of life instrument: Reference data for users. Qual. Life Res. 2002; 11:173-183. [PubMed: 12018740]

2. Heo S, Moser DK, Lennie TA, Zambroski CH, Chung ML. A comparison of health-related quality of life between older adults with heart failure and healthy older adults. Heart Lung. 2007; 36:16-24. [PubMed: 17234473]

3. Alla F, Briancon S, Guillemin F, Juilliere Y, Mertes PM, Villemot JP, Zannad F. Self-rating of quality of life provides additional prognostic information in heart failure. Insights into the EPICAL study. Eur J Heart Fail. 2002; 4:337-343. [PubMed: 12034160]

4. Lewis EF, Johnson PA, Johnson W, Collins C, Griffin L, Stevenson LW. Preferences for quality of life or survival expressed by patients with heart failure. J. Heart Lung Transplant. 2001; 20:10161024. [PubMed: 11557198]

5. Bekelman DB, Havranek EP, Becker DM, Kutner JS, Peterson PN, Wittstein IS, Gottlieb SH, Yamashita TE, Fairclough DL, Dy SM. Symptoms, depression, and quality of life in patients with heart failure. J. Card. Fail. 2007; 13:643-648. [PubMed: 17923356]

6. Blinderman CD, Homel P, Billings JA, Portenoy RK, Tennstedt SL. Symptom distress and quality of life in patients with advanced congestive heart failure. J. Pain Symptom Manage. 2008; 35:594603. [PubMed: 18215495]

7. Albert N, Trochelman K, Li J, Lin S. Signs and symptoms of heart failure: Are you asking the right questions? Am.J. Crit. Care. 2010; 19:443-452. [PubMed: 19940253]

8. Heo S, Doering LV, Widener J, Moser DK. Predictors and effect of physical symptom status on health-related quality of life in patients with heart failure. Am.J. Crit. Care. 2008; 17:124-132. [PubMed: 18310649]

9. Faller H, Stork S, Schuler M, Schowalter M, Steinbuchel T, Ertl G, Angermann CE. Depression and disease severity as predictors of health-related quality of life in patients with chronic heart failure-A structural equation modeling approach. J. Card. Fail. 2009; 15:286-292 e282. [PubMed: 19398075]

10. Parissis JT, Nikolaou M, Farmakis D, Bistola V, Paraskevaidis IA, Adamopoulos S, Filippatos G, Kremastinos DT. Clinical and prognostic implications of self-rating depression scales and plasma B-type natriuretic peptide in hospitalised patients with chronic heart failure. Heart. 2008; 94:585589. [PubMed: 17761502]

11. Hunt SA, Baker DW, Chin MH, Cinquegrani MP, Feldman AM, Francis GS, Ganiats TG, Goldstein S, Gregoratos G, Jessup ML, Noble RJ, Packer M, Silver MA, Stevenson LW, Gibbons RJ, Antman EM, Alpert JS, Faxon DP, Fuster V, Jacobs AK, Hiratzka LF, Russell RO, Smith SC Jr. ACC/AHA guidelines for the evaluation and management of chronic heart failure in the adult: 
Executive summary. A report of the American College of Cardiology/American Heart Association Task Force on Practice Guidelines (Committee to revise the 1995 Guidelines for the Evaluation and Management of Heart Failure). J.Am. Coll. Cardiol. 2001; 38:2101-2113. [PubMed: 11738322]

12. Scott LD. Caregiving and care receiving among a technologically dependent heart failure population. ANS. Adv. Nurs. Sci. 2000; 23:82-97. [PubMed: 11104326]

13. Rector TS, Cohn JN. Assessment of patient outcome with the Minnesota Living with Heart Failure questionnaire: Reliability and validity during a randomized, double-blind, placebo-controlled trial of pimobendan. Pimobendan Multicenter Research Group. Am. Heart J. 1992; 124:1017-1025. [PubMed: 1529875]

14. Heo S, Moser DK, Riegel B, Hall LA, Christman N. Testing the psychometric properties of the Minnesota Living With Heart Failure Questionnaire. Nurs. Res. 2005; 54:265-272. [PubMed: 16027569]

15. Riegel B, Moser DK, Glaser D, Carlson B, Deaton C, Armola R, Sethares K, Shively M, Evangelista L, Albert N. The Minnesota Living With Heart Failure Questionnaire: Sensitivity to differences and responsiveness to intervention intensity in a clinical population. Nurs. Res. 2002; 51:209-218. [PubMed: 12131233]

16. Zambroski CH, Moser DK, Bhat G, Ziegler C. Impact of symptom prevalence and symptom burden on quality of life in patients with heart failure. Eur J Cardiovasc Nurs. 2005; 4:198-206. [PubMed: 15916924]

17. Storch EA, Roberti JW, Roth DA. Factor structure, concurrent validity, and internal consistency of the Beck Depression Inventory-Second Edition in a sample of college students. Depress. Anxiety. 2004; 19:187-189. [PubMed: 15129421]

18. Beck AT, Steer RA, Ball R, Ranieri W. Comparison of Beck Depression Inventories -IA and -II in psychiatric outpatients. J. Pers. Assess. 1996; 67:588-597. [PubMed: 8991972]

19. Frasure-Smith N, Lesperance F, Irwin MR, Talajic M, Pollock BG. The relationships among heart rate variability, inflammatory markers and depression in coronary heart disease patients. Brain. Behav. Immun. 2009; 23:1140-1147. [PubMed: 19635552]

20. Heo S, Moser DK, Chung ML, Lennie TA. Social status, health-related quality of life, and eventfree survival in patients with heart failure. Eur J Cardiovasc Nurs. 2012; 11:141-149. [PubMed: 21071279]

21. Charlson ME, Pompei P, Ales KL, MacKenzie CR. A new method of classifying prognostic comorbidity in longitudinal studies: Development and validation. J. Chronic Dis. 1987; 40:373383. [PubMed: 3558716]

22. Middel B, Bouma J, de Jongste M, van Sonderen E, Niemeijer MG, Crijns H, van den Heuvel W. Psychometric properties of the Minnesota Living with Heart Failure Questionnaire (MLHF-Q). Clin. Rehabil. 2001; 15:489-500. [PubMed: 11594639]

23. Heo S, Moser DK, Riegel B, Hall LA, Christman N. Testing a published model of health-related quality of life in heart failure. J. Card. Fail. 2005; 11:372-379. [PubMed: 15948088]

24. McHorney CA, Ware JE Jr, Raczek AE. The MOS 36-Item Short-Form Health Survey (SF-36):II. Psychometric and clinical tests of validity in measuring physical and mental health constructs. Med. Care. 1993; 31:247-263. [PubMed: 8450681]

25. Conard MW, Heidenreich P, Rumsfeld JS, Weintraub WS, Spertus J. Patient-reported economic burden and the health status of heart failure patients. J. Card. Fail. 2006; 12:369-374. [PubMed: 16762800]

26. Lee DT, Yu DS, Woo J, Thompson DR. Health-related quality of life in patients with congestive heart failure. Eur J Heart Fail. 2005; 7:419-422. [PubMed: 15718183]

27. Littell, RC.; Milliken, GA.; Stroup, WW.; Wolfinger, RD.; Schabenberger, O. SAS for Mixed Models. Cary, NC: SAS Institute Inc.; 2006.

28. Rumsfeld JS, Havranek E, Masoudi FA, Peterson ED, Jones P, Tooley JF, Krumholz HM, Spertus JA. Depressive symptoms are the strongest predictors of short-term declines in health status in patients with heart failure. J. Am. Coll. Cardiol. 2003; 42:1811-1817. [PubMed: 14642693]

29. Koenig HG. Depression in hospitalized older patients with congestive heart failure. Gen. Hosp. Psychiatry. 1998; 20:29-43. [PubMed: 9506252] 
30. Sullivan MJ, Wood L, Terry J, Brantley J, Charles A, McGee V, Johnson D, Krucoff MW, Rosenberg B, Bosworth HB, Adams K, Cuffe MS. The Support, Education, and Research in Chronic Heart Failure Study (SEARCH): A mindfulness-based psychoeducational intervention improves depression and clinical symptoms in patients with chronic heart failure. Am. Heart J. 2009; 157:84-90. [PubMed: 19081401]

31. Todero CM, LaFramboise LM, Zimmerman LM. Symptom status and quality-of-life outcomes of home-based disease management program for heart failure patients. Outcomes Manag. 2002; 6:161-168. [PubMed: 12385167]

32. Muller-Tasch T, Peters-Klimm F, Schellberg D, Holzapfel N, Barth A, Junger J, Szecsenyi J, Herzog W. Depression is a major determinant of quality of life in patients with chronic systolic heart failure in general practice. J. Card. Fail. 2007; 13:818-824. [PubMed: 18068614]

33. Soriano N, Ribera A, Marsal JR, Brotons C, Cascant P, Permanyer-Miralda G. Improvements in health-related quality of life of patients admitted for heart failure. The HF-QoL study. Rev. Esp. Cardiol. 2010; 63:668-676.

34. Faris R, Purcell H, Henein MY, Coats AJ. Clinical depression is common and significantly associated with reduced survival in patients with non-ischaemic heart failure. Eur J Heart Fail. 2002; 4:541-551. [PubMed: 12167395]

35. Carney RM, Blumenthal JA, Stein PK, Watkins L, Catellier D, Berkman LF, Czajkowski SM, O'Connor C, Stone PH, Freedland KE. Depression, heart rate variability, and acute myocardial infarction. Circulation. 2001; 104:2024-2028. [PubMed: 11673340]

36. Corvera-Tindel T, Doering LV, Roper J, Dracup K. Emotional functioning drives quality of life in men with heart failure. Prog. Cardiovasc. Nurs. 2009; 24:2-11. [PubMed: 19261137] 


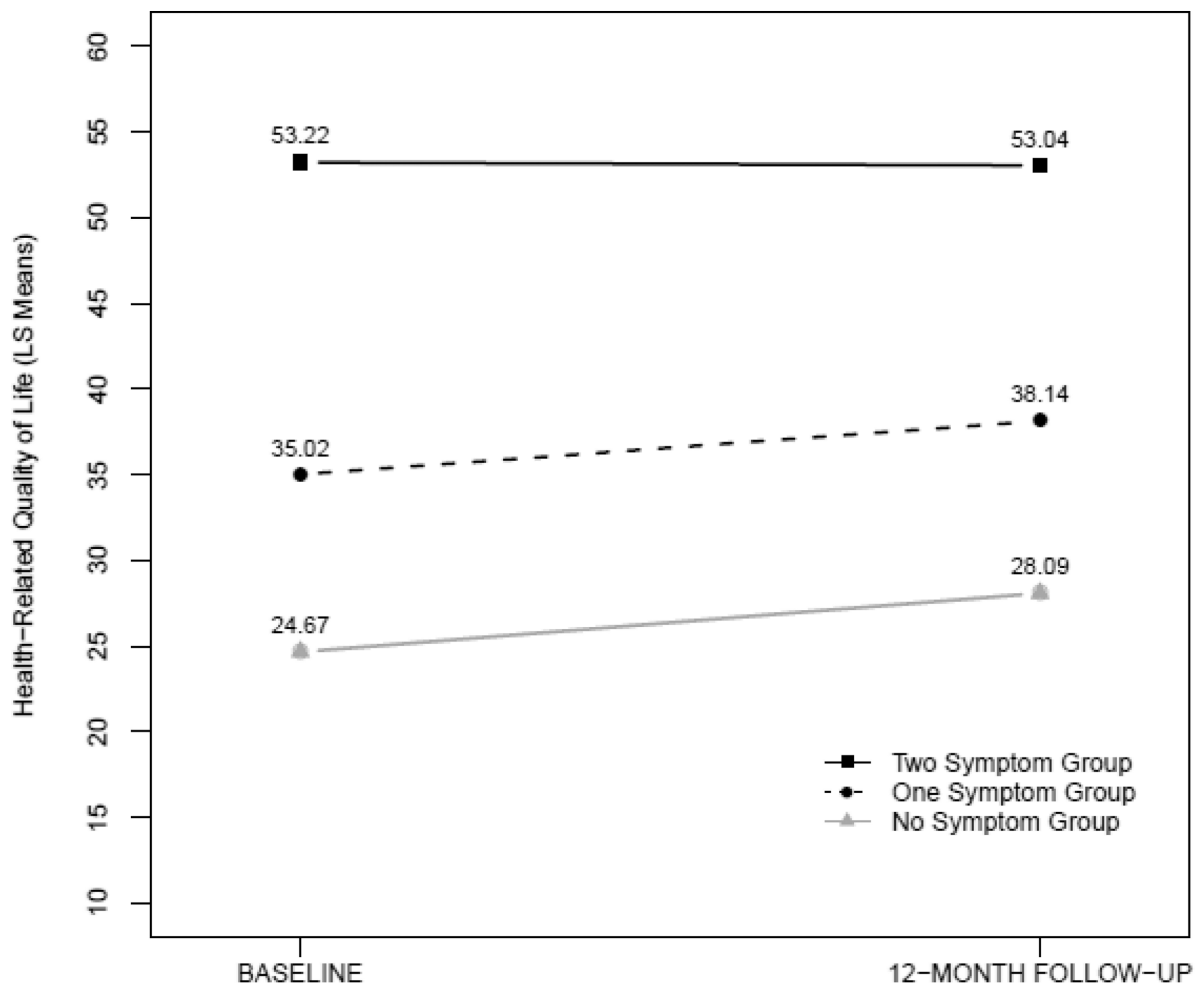

Figure 1.

Health-Related Quality of Life at Baseline and 12-Month Follow-up in the Three Symptom Groups 
Table 1

Comparison of Characteristics and Covariate Status Among Three Symptom Groups

\begin{tabular}{|c|c|c|c|}
\hline Characteristic & $\begin{array}{l}\text { No symptom group } \\
(\mathbf{n}=\mathbf{2 5})\end{array}$ & $\begin{array}{l}\text { One symptom group } \\
\qquad(\mathrm{n}=138)\end{array}$ & $\begin{array}{c}\text { Two symptom group } \\
(\mathrm{n}=61)\end{array}$ \\
\hline \multicolumn{4}{|l|}{ Demographic Characteristics } \\
\hline Age (years), Mean $( \pm \mathrm{SD})^{a}$ & $64.9( \pm 12.3)$ & $63.1( \pm 11.7)$ & $57.3( \pm 9.9)$ \\
\hline Male, N (\%) & $20(80.0)$ & $88(63.8)$ & $43(70.5)$ \\
\hline Married, N (\%) & $14(56.0)$ & $63(45.7)$ & $31(50.8)$ \\
\hline Caucasian, $\mathrm{N}(\%)$ & $13(52.0)$ & $98(71.0)$ & $45(73.8)$ \\
\hline Education level (years), Mean $( \pm \mathrm{SD})^{a}$ & $14.4( \pm 2.8)$ & $14.3( \pm 3.3)$ & $12.7( \pm 2.5)$ \\
\hline \multicolumn{4}{|l|}{ Financial status, $\mathrm{N}(\%)^{a}$} \\
\hline Income $<$ demand & $6(24.0)$ & $28(20.3)$ & $28(45.9)$ \\
\hline Income $=$ demand & $11(44.0)$ & $69(50.0)$ & $30(49.2)$ \\
\hline Income $>$ demand & $8(32.0)$ & $41(29.7)$ & $3(4.9)$ \\
\hline \multicolumn{4}{|l|}{ Clinical Characteristics } \\
\hline Comorbidities, Mean $( \pm \mathrm{SD})^{b}$ & $2.8( \pm 2.0)$ & $2.8( \pm 1.6)$ & $3.4( \pm 2.1)$ \\
\hline Left Ventricular Ejection Fraction (\%), Mean $( \pm$ SD) & $34.6( \pm 13.4)$ & $34.5( \pm 13.7)$ & $34.8( \pm 13.4)$ \\
\hline Etiology (ischemic), N (\%) & $10(40.0)$ & $64(46.4)$ & $27(44.3)$ \\
\hline NYHA functional class (Class III \& IV), N (\%) ${ }^{a}$ & $2(8.0)$ & $52(37.7)$ & $32(52.5)$ \\
\hline Diabetes mellitus, $\mathrm{N}(\%)$ & $10(40.0)$ & $44(31.9)$ & $25(41.0)$ \\
\hline Hypertension, $\mathrm{N}(\%)$ & $18(72.0)$ & $88(63.8)$ & $46(75.4)$ \\
\hline Angiotensin converting enzyme inhibitors, $\mathrm{N}(\%)$ & $17(68.0)$ & $92(66.7)$ & $38(62.3)$ \\
\hline Beta-blockers, $\mathrm{N}(\%)$ & $21(84.0)$ & $124(89.9)$ & $51(83.6)$ \\
\hline \multicolumn{4}{|l|}{ Health perception, $\mathrm{N}(\%)^{a, c}$} \\
\hline Excellent & $2(8.0)$ & $5(3.6)$ & $0(0.0)$ \\
\hline Very good & $10(40.0)$ & 15 (10.9) & $3(4.9)$ \\
\hline Good & $10(40.0)$ & $62(44.9)$ & $9(14.8)$ \\
\hline Fair & $3(12.0)$ & 45 (32.6) & $33(54.1)$ \\
\hline Poor & $0(0.0)$ & $11(8.0)$ & $16(26.2)$ \\
\hline
\end{tabular}

NYHA $=$ New York Heart Association.

$a_{\mathrm{p}<.05 .}$

${ }^{b}$ Analysis based on Kruskal-Wallis (group 1 median = 2; group 2 median = 3; group 3 median = 3). 
${ }^{c}$ Analysis based on exact chi-square. 